\title{
POLÍTICA Y GEOPOLÍTICA EN EL PROCESO DE REGIONALIZACIÓN DEL REGIMEN AUTORITARIO CHILENO (1973-1990)
}

\author{
Rodolfo Quiroz \\ USP - Universidade de São Paulo \\ Universidad Alberto Hurtado - Chile
}

\section{Resumen}

A partir de las profundas transformaciones económicas y los cambios institucionales vividos en Chile entre 1973-1990, se reflexiona el carácter geopolítico del proceso de regionalización territorial efectuada por el régimen autoritario. Se discuten los sentidos políticos y geopolíticos de la regionalización como parte del proyecto hegemónico del régimen, en el cual la regionalización cumple una función fundamentalmente políticasocial, proyectando la lógica de poder del Estadoterritorial. Lejos de una geopolítica tradicional ligada a tareas estrictamente militares, se analiza la regionalización en términos de verticalidad, persistencia institucional y vigilancia política. Se enfatiza así que dichos elementos marcan un tipo de estrategia geopolítica y una regionalización política que, acorde a las nuevas transformaciones económicas del periodo, son también funcionales a un principio de la geopolítica clásica: el Estado sobre la sociedade.

Palabras clave: Regionalización; Geopolítica; Hegemonía.

\begin{abstract}
Resumo
A partir das profundas transformações econômicas e das mudanças institucionais acontecidas no Chile entre 1973-1990, se reflete sobre o caráter geopolítico do processo de regionalização territorial efetuada pelo regime autoritário. Se discutem os sentidos políticos e geopolíticos da regionalização como parte do projeto hegemônico do regime, no qual a regionalização cumpre uma função fundamentalmente política-social, projetando a lógica de poder do Estado-territorial. Longe de uma geopolítica tradicional associada às tarefas estritamente militares, se analisa a regionalização em termos de verticalidade, persistência institucional e vigilância política. Se enfatiza assim que ditos elementos marcam um tipo de estratégia geopolítica e uma regionalização política que, em consonância às novas transformações econômicas do período, são também funcionais a um princípio da geopolítica clássica: o Estado acima da sociedade.
\end{abstract}

Palavras-clave: Regionalização, Geopolítica, Hegemonia.

\section{Introducción}

A

probada en julio de 1974, la regionalización territorial del país fue una de las primeras reformas estructurales imprimidas por el régimen autoritario. Lejos de ser una obra momentánea o episódica, la racionalización y el perfeccionamiento de los recortes territoriales fue 
una preocupación permanente del régimen autoritario ${ }^{1}$. En términos estructurales la reforma contempló, entre otros asuntos, una nueva división política-territorial, una nueva estructura jerárquica de autoridades en cuatro niveles (nacional, regional, provincial y comunal), además de un nuevo modelo de administración pública del Estado regional y municipal, todo ello, en medio de profundas e inéditas transformaciones económicas que pusieron a Chile como el laboratorio del neoliberalismo a nivel internacional (GARATE, 2012).

Pese a la amplitud de sus significaciones, por lo general, los estudios sobre el periodo autoritario no profundizan la relación entre el proyecto del régimen autoritario y los contenidos y formas de la regionalización, remitiéndose a solo tres aspectos fundamentales: primero, se subraya el aspecto "exitoso" de la reforma en tanto racionalidad burocrática weberiana, legitimada por su legalidad y persistencia en el tiempo (BOISIER, 2000 y 2010; JORDAN y VILLARROEL, 1990); segundo, se destaca la contradicción entre descentralización política y centralismo, puesto que la regionalización fue una desconcentración administrativa del Estado y no una distribución del poder central (VALENZUELA, 2015); y, por último, se defiende un fundamento geopolítico reflejado en el recorte espacial de las regiones y la prioridad de recursos y políticas en las regiones extremas en una lógica de seguridad nacional (ARENAS, 2009; JORDAN y VILLARROEL, 1990).

Sin desconocer estas contribuciones, nuestra investigación busca enfatizar un camino diferente y poco explorado. Es decir, más allá de la racionalidad burocrática y la intensificación del centralismo, consideramos que el núcleo central para comprender la regionalización se fundamenta en la disputa por la matriz política del Estado chileno (LIRA, 2004). Por tanto, es a partir de esa jerarquía política que debe ser analizada la regionalización, tanto en sus rendimientos geopolíticos, tanto en las contradicciones de las relaciones sociales y de poder que emergen de sus recortes y contenidos. Esto implica, entre otros asuntos, que la regionalización no es hegemónica en sí misma, sino que siempre forma parte de un proyecto hegemónico que le da forma y contenido, espacialidades y tiempos. Y no puede comprenderse sin esa posición estratégica y movediza dentro del régimen autoritario chileno y su búsqueda profunda por alterar el régimen político y económico tradicional.

En este contexto, la geopolítica instalada en el proceso de regionalización chilena debe comprenderse por su capacidad de "construir sua unidade nacional interna do ponto de vista da organização política do território, e de transformar esse dado em poder de Estado, a fim de projetá-lo na sua política externa" (COSTA, 2013, p. 23). Lejos de un conjunto estático de principios abstractos y rígidos, más bien, la geopolítica del régimen chileno se expresó en un conjunto de técnicas de aplicación que buscó dar coherencia y cohesión al proyecto político y económico del régimen autoritario, a través de una nueva división política del territorio chileno y una serie de políticas públicas asociadas a su institucionalización. Puesta en movimiento, así, la geopolítica está anclada a una gestión estratégica del territorio definido por los intereses del régimen autoritario. Como señala Berta Becker, podemos definir la gestión como "a prática estratégica científicotecnológica que dirige, no tempo, a coerência de múltiplas decisões e ações para atingir uma finalidade" (BECKER, 2012, p. 128). De tal manera, la geopolítica de la regionalización se definió bajo principios económicos y prácticos, basados en la realidad política y social del país andino,

\footnotetext{
${ }^{1}$ Hasta los últimos años del régimen autoritario (1985-1990), se elaboraron y proyectaron recortes territoriales - nuevos límites de provincias y comunas -, gestionando importantes cambios institucionales destinados a fortalecer la regionalización.
} 
disponiéndose no solo para absorber conflictos sino también para formular grandes maniobras e instrumentos técnicos que posibilitaran la vigencia de la autoridad cívico-militar de la época. Se trató, pues, de una gestión científica-tecnológica del poder autoritario que "instrumentalizou o saber de direção política, de governo", permitiéndole una mayor logística "no sentido da poderosa preparação de meios e da velocidade de sua atuação, referente esta não só à rapidez como à projeção para o futuro" (BECKER, 2012, pp. 128-129).

De ahí que nuestra tesis propone que, lejos del pensamiento desarrollista de los círculos militares de la época, la regionalización impulsada por el alto mando militar chileno permitió, finalmente, re-politizar la influencia del régimen autoritario en la sociedad chilena, fortaleciendo su pretensión hegemónica. Siguiendo a Sevilla (2014) entendemos hegemonía territorial como aquella "práctica de diseminación de formas de uso y concepción del espacio - de códigos que territorializan ese espacio - compatibles con el proyecto de orden socioespacial del bloque dominante" (SEVILLA, 2014, p. 63). La hegemonía, entonces, puede constituirse como a) "un principio de dirección en toda una serie de ámbitos (económico, intelectual, moral, etc.) que permite a un determinado bloque guiar la sociedad a través del consenso y sin imposiciones violentas, lo que garantiza un orden político estable y duradero" (SEVILLA, 2014, p. 54); b) al mismo tiempo, la hegemonía es difusa y se extiende ampliamente en el conjunto del tejido social, circulando a diferentes niveles y dinámicas, de lo micro a lo macro; c) "Toda relación hegemónica es necesariamente una relación pedagógica" (SEVILLA, 2014, p. 55); d) "Transmite una concepción del mundo que se integra en las conciencias como sentido común y se traslada al nivel de lo vivido en las prácticas materiales y el lenguaje" (ídem); e), por último, el propósito central de la hegemonía es legitimar "un determinado proyecto de orden social, facilitando la labor del gobierno mediante la producción de consenso y reduciendo por tanto la necesidad de coerción y uso de la fuerza" (ídem).

\section{Qué geopolítica para qué regionalización}

Establecidas estas consideraciones entramos al problema hegemónico de la regionalización chilena y sus desdoblamientos centrales. ¿Qué fundamento geopolítico expresó el recorte de las regiones y qué tipo de geopolítica fue colocada en el proceso de regionalización? Conviene destacar, de entrada, que la regionalización del periodo no se fundamentó en una posición "filosófica" o corriente política-militar exclusiva, sino más bien, representó una combinación selectiva de enfoques: desarrollismo, nacionalismo, conservadurismo, liberalismo, geografía física, militarismo, etc. Es decir, se fue construyendo y validando en el proceso mismo de su implementación, mediante una rearticulación por etapas y prioridades, que implicó diferentes escalamientos del poder del Estado y alcances político-sociales (LIRA, 2004; CHATEAU, 1978).

Ahora bien, pese la amplitud y diversidad de prácticas institucionales que movilizó, la regionalización permanentemente proyectó un determinado escenario político: Pinochet como gobernador de la patria y el régimen autoritario como una alternativa real de gobierno que podría trascender y permanecer en el tiempo irrevocablemente. Fue en este sentido que se alteró el antiguo sistema territorial y, por lo tanto, el conjunto de prácticas públicas del régimen en su dimensión político estatal.

El primer recorte espacial del periodo autoritario, el decreto ley $n^{\circ} 575$ que dio forma a la nueva división político-administrativa de Chile, se aprobó en julio de 1974, a solo diez meses de 
inicio del régimen. La decisión final de la regionalización fue tomada por la Junta de Gobierno², previo al informe elaborado por la Comisión Nacional de Reforma Administrativa y Regionalización (CONARA). Meses antes, la CONARA recibió dos proposiciones alternativas de regionalización que, posteriormente, modificó, presentando un tercer modelo regional al informe ${ }^{3}$. Comparadas las propuestas, la Junta de Gobierno reformuló y aprobó la regionalización, estableciendo trece nuevas unidades político-administrativas subnacionales, es decir, se pasó de un país dividido en veinticinco provincias a uno de doce regiones más un Área Metropolitana de Santiago, que internamente se constituían de provincias y comunas ${ }^{4}$. A pesar de presentarse como una regionalización de nuevo tipo (nuevos criterios modernos de dotación de recursos naturales, estructura urbanorural garante de un nivel de servicios básico, base poblacional suficiente, seguridad nacional en armonía con metas de desarrollo regional y nacional, eficiencia de la administración territorial), el recorte regional expresó importantes similitudes espaciales con la sub-división de macro-zonas elaborado por la Oficina de Desarrollo y Planificación Nacional (ODEPLAN) de 1969, aprobada bajo el gobierno de Eduardo Frei Montalva ${ }^{5}$ (1964-1970).

En las regiones extremas el recorte continuo con la misma escala espacial de las antiguas provincias, erigido en la Constitución de 1925, al tiempo que se mantuvo el mismo criterio de agrupación por provincias de la ODEPLAN. Los principales cambios y modificaciones de fronteras internas se articularon en la zona centro-sur del país en concordancia a la mayor densidad poblacional. Estas nuevas regiones intentaron impulsar un sentido modernizador del Estado, siendo nombradas por números romanos de norte a sur, un hecho que ya había sido colocado por el equipo de ODEPLAN cinco años antes del golpe de Estado. De esta forma, todo indica que el primer recorte regional implementado por la Junta Militar se ejecutó sobre una base espacial ya consensuada, la cual habría sido cimentada por la ODEPLAN (1964-1970).

Por tanto, no es el recorte espacial de las regiones lo que definió estrictamente el carácter geopolítico de la regionalización, en una primera instancia. A nuestro juicio la particularidad geopolítica en la regionalización chilena se profundizó en la permanencia, vigilancia y distribución del poder vía unidades territoriales: un reforzamiento de la autoridad estatal. En efecto, la regionalización de una u otra forma debió construir una imagen de futuro, una imagen donde la geopolítica del orden tuviese un lugar central.

Si bien los sectores militares promovieron un diagnóstico geográfico económico y físico de todo el territorio del país - que se iba a enfrentar mediante tres objetivos centrales ${ }^{6}$-, en la prácti-

\footnotetext{
${ }^{2}$ La Junta de Gobierno fue el máximo órgano ejecutivo del régimen autoritario y era constituida por los tres comandantes de las FFAA chilenas y el Director General de Carabineros pero, en última instancia jerárquica, era presidida por el general de Ejército Augusto Pinochet.

${ }_{3}^{3}$ La primera alternativa fue presentada por el equipo regional de la Oficina de Desarrollo y Planificación Nacional (ODEPLAN) y se componía de diez regiones; la segunda propuesta fue elaborada por el Comité Interdisciplinario de Desarrollo Urbano (CIDU) de la Pontificia Universidad Católica de Chile y contemplaba cinco macro-regiones; la tercera propuesta fue presentada por la CONARA tras una revisión de ambas propuestas anteriores, contemplando once nuevas regiones.

${ }^{4}$ A nivel territorial nacional Chile tiene cuatro niveles jerárquicos de mayor a menor escala: nacional, regional, provincial y comunal.

${ }^{5}$ El decreto ley $n^{\circ}$ 1104, promulgado por el gobierno de Eduardo Frei Montalva, el 19/08/1969, estableció que el Estado de Chile agrupó sus provincias, creando diez regiones de planificación económicas más una Zona Metropolitana (la de Santiago).

${ }^{6}$ 1) superar los graves problemas de desvinculación geográfica y vastos espacios vacíos -fronteras interiores- que atentaban contra la Seguridad Nacional; 2) reducir las desigualdades regionales marcadas por una "macrocefalia metropolitana" y "la generación de cordones de miseria alrededor de los grandes centros urbanos" (CANESSA, 1982, p. 21); 3) proyectar un uso racional de los recursos estratégicos en cada una de las regiones (ARENAS, 2007).
} 
ca, la regionalización no disminuyó las desigualdes entre la Región Metropolitana y el resto de las regiones (JORDAN y VILLARROEL, 1990; APEY, 1983; RIFFO, 2005) ni tampoco fortaleció el uso estrátegico de los recursos naturales como una política soberana. No obstante, sí tuvo un efecto concreto: robusteció al poder central, convirtiéndolo, según Chateau, "en una herramienta de mayor eficiencia" (CHATEAU, 1978, p. 202).

\section{Institucionalidad, permanencia y regionalización}

De esta forma, el cáracter geopolítico de la regionalización se expresó, en primer lugar, en la permanencia institucional del poder autoritario-territorial: un nuevo tejido de Estado. Junto con la aprobación de la división territorial político-administrativa y el Sistema de Gobierno Interior, se crearon diferentes instituciones y dispositivos técnicos destinados a dar permanencia al proceso de regionalización, es decir, una regionalización capaz de trascender más allá de gobiernos y coyunturas con una estricta jerarquía al interior del funcionamiento del Estado territorial.

Este proceso de institucionalización desde su inicio se destaca por la creación de la CONARA en 1973 y sus nuevas funciones y jerarquías conforme al avance de la regionalización. Posteriormente, 1975, se creó el Fondo de Desarrollo Regional (FNDR, 1975) con el propósito de apoyar obras de infraestructura con impacto económico y otorgar un debido financiamiento a la nueva estructura regional del país; mismo propósito que va ser reforzado, en 1979, con la creación del Fondo Común Municipal (FCM) para los gobiernos municipales. El nuevo Sistema de Gobierno también contempló la desconcentración de los Ministerios de Educación, Salud, Vivienda y Urbanismo en términos regionales "bajo el principio de que la administración central no debe realizar ninguna acción que pueda cumplir eficientemente la administración regional" (CANESSA, 1982, p. 19). Se crearon así las Secretarías Regionales Ministeriales (SEREMI'S) que debían reforzar la gestión del Intendente en las áreas particularmente sociales. En 1975 fue el turno de las Secretarías Regionales de Planificación y Coordinación (SERPLAC), siendo oficinas técnicas de apoyo directo a los intendentes, las cuales dieron la pauta para la creación de las Secretarías Comunales de Planificación (SECPLAC) que definen la asesoría técnica de los alcaldes. En una lógica de integración de la comunidad, pero en última jerarquía institucional, el Sistema contempló dos instancias participativas: los Consejos Regionales de Desarrollo (COREDE) y los Consejos de Desarrollo Comunal (CODECO), ambos dispositivos debían expresar las percepciones e inquietudes de la comunidad regional o comunal.

La lógica geopolítica que dominó la creación de estas institucionalidades se definió bajo dos orientaciones fundamentales: permitir una mayor "agilización de trámites burocráticos" (CHATEAU, 1978, p. 236) y reducir las "tareas del aparato estatal en el cambio de orientación económica requerida por el modelo de mercado" (ídem). De este modo, la regionalización no intervenía en las dinámicas propiamente productivas y se dedicaba exclusivamente de cautelar "el diseño y control de las grandes orientaciones políticas" (CHATEAU, 1978, p. 235), creando una "mayor eficiencia en el ejercicio del poder" y cohesión entre unidades regionales y niveles centrales.

Iniciada la década del ochenta se crearon las Universidades Regionales que, entre otras dinámicas, fortalecieron la influencia de la regionalización en términos de ciencia y educación mediante 22 universidades antiguamente dependientes de la Universidad Técnica del Estado y la 
Universidad de Chile. Finalmente, en 1985, se creó la Subsecretaría de Desarrollo Regional (SUBDERE) con el fin de cautelar los contenidos de la regionalización al interior del Estado. Se localizó institucionalmente al interior del Ministerio del Interior, la estructura más centralizada y jerárquica del Estado después de la Presidencia de la República. Un hecho que no puede ser omitido pues representa, a nuestro juicio, el sentido estratégico de la geopolítica al interior de la regionalización: proyectar el Estado territorial como una forma de gobierno sin procesos de deliberación democrática y anclada a la máxima jerarquía política del poder centralizado.

\section{Una logística autoritaria y estratégica: verticalidad de mando}

Empujada una y otra vez por diferentes mecanismos institucionales, la geopolítica se cristalizó en el funcionamiento interno de la regionalización. Antiguamente la máxima autoridad a nivel subnacional, el Intendente provincial, carecía de posibilidades de gestión político-territorial, remitiéndose a tareas de orden administrativas signadas por el poder ejecutivo. La nueva regionalización del país modificó esta dinámica pues reforzó no solo la capacidad de gestión de las autoridades regionales, sino que también implementó una infraestructura estatal de gobierno regional y municipal con mayores recursos y atribuciones que, lejos de influenciar o democratizar el poder del régimen, permitió una mejor "dirección y control" del poder central sobre las unidades territoriales (CHATEAU, 1978).

Este efecto se explica por razones. Primero, el cambio de 25 intendentes provinciales a uno de 13 Intendentes regionales permitió un mayor seguimiento y monitoreo de la autoridad central a las regiones (CHATEAU, 1978). Segundo, los nuevos intendentes tenían mayores atribuciones y recursos para construir una gestión de gobierno regional. Sin embargo, dichas funciones se limitaban a ámbitos particulares o administrativos, puesto que la definición política era previamente establecida por el poder central. Este elemento permitía que el poder central pudiese enfocarse exclusivamente en la elaboración de políticas estructurales que debían ser aplicadas en todo el país, ganando tiempo y energía (CHATEAU, 1978, p. 203). Tercero, todas las autoridades territoriales del nuevo Gobierno Interior (Intendente para la región, Alcalde para la comuna y Gobernador para la provincia) eran designadas y dependían directamente de la confianza del General Pinochet, generando así "una sola línea de verticalidad de mando" (CHATEAU, 1978, p. 203). Misma concentración de poder se repetía a las autoridades regionales, rectores de las universidades, secretarios regionales y directores de servicios públicos, todos jerárquicamente dependientes de la estructura central localizada en Santiago.

De esta forma, la geopolítica incorporada en la regionalización cautelaba un particular tipo de funcionamiento político en las unidades territoriales, una jerarquía de mando que se desplegaba logísticamente en todo el territorio nacional. Ahora bien, ¿cómo aplicar una regionalización celosa del poder de la autoridad central, técnicamente jerarquizada, pero al mismo tiempo, inserta en las comunidades territoriales? En palabras del general Canessa la regionalización implicó "una nueva modalidad en el ejercicio del poder, ya que la autoridad central, anteriormente aislada e invisible, se acerca a la comunidad en todo el ámbito territorial del Estado, hace sentir su presencia efectiva y adquiere un conocimiento directo de las aspiraciones e inquietudes de todos los ciudadanos" (CANESSA, 1982, p. 16). 


\section{Recortes espaciales, comunas y geopolítica}

En efecto, si durante los primeros años los recortes espaciales estuvieron centrados en el reforzamiento y la implementación del sistema político-administrativo en la escala regional y provincial, finalizada la década del setenta y principios del ochenta, las principales modificaciones se centraron en la esfera comunal. Para el general Canessa esto se explicaba porque la comuna era:

La unidad territorial básica de todo el proceso, donde debe darse en forma plena la participación de la comunidad y en que la acción gubernamental se hace presente en la forma más directa a cada uno de sus integrantes. Su ordenación territorial se basa en las condiciones tipológicas, su naturaleza económica-social, en el hábitat y en su rango administrativo. Debe permitir una adecuada organización social de la población y eficiente prestación de servicios locales, ordenación y equipamiento de su territorio. En resumen, es el órgano final de la jerarquía administrativa y territorial del Estado, constituyendo el nivel político y administrativo más directo de contacto de la comunidad organizada con sus gobernantes (CANESSA, 1982, p. 23).

En 1979 se oficializó el primer recorte de límites comunales, junto con un segundo ajuste de las provincias, exceptuando la Región Metropolitana, creándose cuatro nuevas provincias (Parinacota, Cardenal Caro, Cauquenes y Coyhaique) y quince nuevos municipios en todo el país. Estos recortes aumentaron la presencia del Estado en el territorio: ya sea por la construcción material de oficinas y puestos de trabajo públicos, ya sea por la nominación de autoridades para el ejercicio de gobierno interior. En los años sucesivos, 1980 y 1981, se entregó un segundo recorte espacial de provincias y comunas de la Región Metropolitana, más un nuevo ajuste comunal por regiones ${ }^{7}$ excluyendo la Región de La Araucanía. Estos recortes se aprobaron en tiempos de una agitada transformación económica y social puesto que comenzaban las reformas neoliberales a nivel de ventajas comparativas, al tiempo que emergían las primeras protestas urbanas y populares contra el régimen autoritario tras la recesión económica en 1982. Estas transformaciones y movimientos en el campo urbano van a ser expresadas mediante el Plan Intercomunal de Santiago y su Ordenanza de 1979, que liberalizó importantes áreas rurales para el uso urbano y un complejo programa de erradicaciones y radicaciones de sectores populares que serán reubicados en las periferias del gran Santiago. La Región Metropolitana fue el máximo laboratorio de experimentación comunal en materia de regionalización: se crearon 17 nuevas comunas en 1981, junto con la creación de una nueva provincia y un reglamento específico de Gobierno y Administración Metropolitano para Santiago.

\section{Control, autoridad y vigilancia en los espacios de representación}

¿Cual es la geopolítica que se juega en estos recortes espaciales? En el caso de la Región Metropolitana se trata de 17 nuevas instalaciones públicas, funcionales al régimen, en un ambiente de alta confusión social - recordemos que las protestas iniciadas en 1983 no se detuvieron hasta

\footnotetext{
${ }^{7}$ Antofagasta, Tarapacá, Atacama, Coquimbo, Magallanes, Libertador General Bernardo O’Higgins, Aysén, Bio-Bio, El Maule, Valparaíso, Los Lagos.
} 
el término del régimen autoritario -. Los recortes espaciales de comunas se inscribieron dentro de un rígido cuadro institucional que suponía la separación de un poder político, propio de la autoridad militar por arriba, en la cabeza del Estado. Y la existencia de un poder social de base, asociado a las organizaciones comunitarias, gremios y colegios profesionales, ubicados en el nivel más local de la sociedad civil:

el poder social - argumentaba la Junta en 1974 - está llamado a convertirse en el cauce orgánico más importante de expresión ciudadana. Chile tiene una larga tradición de organización social, que se remonta a su origen hispánico. Los cabildos, la comuna autónoma, el sindicalismo laboral y el gremialismo extendido a todo nivel son hitos de un proceso que revela que el pueblo chileno ha estado permanentemente renovando sus formas de organización social de acuerdo con su evolución histórico-social. Es imperioso restituir a los Municipios el papel trascendental e insustituible que les corresponde como vehículo de organización social, devolviéndoles su carácter de entidad vecinal al servicio de la familia y del hombre, incompatible por tanto con la politización a la cual se los ha arrastrado (JUNTA DE GOBIERNO, 1974).

En efecto, la operación geopolítica de pensar nuevas comunas justamente se inscribió en una lógica de construir un mayor control del espacio comunitario y ampliar la influencia del régimen en la sociedad (CHATEAU, 1977, p. 177). Fue una hipótesis política que proyectó un espacio social funcional al régimen, creando una posición territorial de Estado que, desde la primera línea del comando central, estableció condiciones de integración de la comunidad para el programa de gobierno y su vigilancia. Las acciones políticas municipales, desde luego, reflejaron el arsenal político, técnico y programático del régimen autoritario, conforme al conjunto del sistema de control que imprimió la regionalización. Se trató, pues, de "proveer una estructura que permita rehacer ciertas líneas de comunicación con la población y eventualmente de participación de ella" (CHATEAU, 1978, p. 236). Tanto las regiones como las comunas se transformaron en los espacios de representación disponibles para la sociedad civil en un contexto de fuerte autoritarismo institucional. De ahí la importancia de promover diferentes dispositivos de participación que, junto con realzar la identificación patriótica y desmantelar la idea de representación política de los partidos tradicionales, permitieran, sobre todo, delinear una propia idea de democracia del régimen. Una "nueva" democracia o "democracia protegida" (HUNEEUS, 2016) con sentido de identificación comunal o regional, pero siempre subordinada a la autoridad estatal y fuera de posibles conflictos marcados por la estructura económica o de clases.

Es por estas razones que la regionalización también implicó la creación de importantes programas y proyectos de orden social destinados a recrear lazos entre comunidades regionales y comunales: fortalecimiento de campeonatos de fútbol regional, concursos de reina de belleza, fortalecimiento de colegios profesionales y organizaciones gremiales, creación de centros de madres, clubes deportivos y ancianos, todos dispositivos destinados a llenar de contenido el proyecto de regionalización y la oferta de gobernabilidad del régimen: una "nueva" democracia tecnificada formada por gremios empresariales y organizaciones comunitarias funcionales al régimen que aparentaban representar la voluntad popular. Estas líneas de participación, en efecto, si bien integraban a la comunidad en ciertos programas y planes de orden "apolíticos", por sobre todo, influenciaban y vigilaban la efectiva asimilación de los principios colocados por el régimen. Se trata- 
ba de una participación panóptica pues, desde la propia escala geográfica de comunas o regiones, se institucionalizaba un orden autoritario y un determinado tipo de participación que legitimaba la verticalidad de mando en el proceso de regionalización.

Como cualquier otra estrategia geopolítica de control, en efecto, la participación técnicamente no podía movilizar demandas estructurales ya que, lo que se entendía como participación, debía devolver "su carácter de entidad vecinal al servicio de la familia y el hombre" (JUNTA DE GOBIERNO, 1974). No obstante, de una u otra forma, las líneas de participación permitían establecer un vínculo con la población, ampliando la autoridad territorial, sobre todo en aquellas territorialidades históricamente aisladas y excluidas donde la única posibilidad de integración recaía en el Estado: caso de la región Austral.

\section{Consideraciones finales}

Vigilar, integrar, permanecer. Estas fueron las tres lógicas estructurales que integraron la disputa hegemónica de la regionalización del periodo autoritario. Pensadas desde un punto de vista geopolítico y sin un orden cronológico, ya que son simultáneas y entrelazadas unas de otras, caracterizan también tres tipos de operaciones de poder territorial. Primero, se trató de vigilar y controlar a la población mediante el reforzamiento de nuevas unidades territoriales y la creación de un nuevo sistema de gobierno interior, reforzando una logística política plenamente jerarquizada, en todo el territorio nacional, pero con sofisticados mecanismos de gestión y cohesión en las escalas locales e intermedias. Segundo, a partir del control logístico de todo el territorio nacional y la promoción de autoridades territoriales con plenas facultades de gestión, se pretendió integrar y rehabilitar espacios de representación social, regionales y comunales, anclados fundamentalmente a tres principios básicos: participación subordinada a la autoridad política estatal, resguardo a la propiedad privada y promoción de actividades comunitarias regionales y comunales fuera del régimen deliberativo. Tercero, se buscó dar permanencia al nuevo modelo territorial vía dispositivos jurídicos y, sobre todo, mediante la creación de diferentes institucionalidades al interior del Estado que permitieron cautelar los principios y jerarquías de la regionalización: proyectar el Estado territorial como una forma de gobierno sin procesos de deliberación democrática. El momento paradigmático de este proceso será en 1985, cinco años antes de la finalización del régimen, con la creación de la SUBDERE, institución que, dicho sea de paso, sigue vigente a la fecha.

En otras palabras, existe una plena concordancia entre el proyecto de gobierno del régimen autoritario, la regionalización y el pensamiento geopolítico. Esa concordancia radica en el "deber ser del Estado" (CHATEAU, 1978), siendo la regionalización un refugio del proyecto de poder nacional del régimen autoritario: su búsqueda por hegemonía territorial. La regionalización así otorgó un sentido proyectual a un régimen autoritario que recreó su propia idea de democracia. Su función política básica fue facilitar la implementación orgánica de las autoridades civilesmilitares al interior del Estado y contribuir al desmantelamiento de las expresiones políticas, históricas, disidentes, tradicionales a nivel de Estado-territorial: es decir, constituyó un elemento orgánico de control y vigilancia sobre la comunidad nacional, negando técnicamente cualquier tentativa o resquicio de representación popular o forma democrática opositora. 
La particularidad de la regionalización chilena, en definitiva, es que transitó entre el autoritarismo y el neoliberalismo. Es decir, por un lado, reforzó el control político-técnico sobre las nuevas unidades territoriales. Por el otro, paulatinamente, condicionó vigilancia y legitimidad a la privatización general de redes e infraestructuras territoriales, mediante un estricto sistema de comando y jerarquías territoriales.

Aprovado pela Comissão Científica do III CONGEO - Congresso Brasileiro de Geografia Política, Geopolítica e Gestão do Território

Universidade Federal Fluminense - Niterói (RJ), 2018 
Referencias

APEY, Alfredo. El proceso de reestructuración económica nacional y su impacto en el sistema regional: 1976-1981. Informaciones geográficas, Universidad de Chile, n. 30, pp. 83-97, 1983.

ARENAS, Federico. El Chile de las regiones: una historia inconclusa. Estudios Geográficos, Madrid, V. LXX, n. 266, p. 11-39, 2009.

BECKER, Bertha. A geografia e o resgate da geopolítica. Espaço Aberto, Rio de Janeiro, V. 2, n. 1, p. 117-150, 2012.

BOISIER, Sergio. Chile: la vocación regionalista del gobierno militar. EURE, Santiago, v. 26, n. 77, 2000.

Territorio, estado y sociedad en Chile: la dialéctica de la descentralización - Entre la geografía y la gobernabilidad. Santiago: Mago, 2010.

CANESSA, Julio. Visión geopolítica de la regionalización. Seguridad Nacional, Santiago, n. 24, p. 13- 31, 1982.

COSTA, Wanderley Messias da. Geografia Política e Geopolítica. São Paulo: Editorial USP, 2013.

CHATEAU, Jorge. Geopolítica y regionalización: algunas relaciones. Santiago: Flacso, 1978.

CHILE. Junta de Gobierno. Declaración de principios del gobierno de Chile. Santiago: Editora Nacional Gabriela Mistral, 1974a.

. Ministerio del Interior. Decreto Ley n. 573, de 12 de julio de 1974. Estatuto del gobierno y administración interiores del Estado. Diario Oficial de la República de Chile. Santiago: Biblioteca del Congreso de Chile, 1974b.

Ministerio del Interior. Decreto Ley n. 575, de 13 de julio de 1974. Regionalización del país. Diario Oficial de la República de Chile. Santiago: Biblioteca del Congreso de Chile, 1974c.

COMISIÓN NACIONAL DE LA REFORMA ADMNISTRATIVA. Chile hacia un nuevo destino: su reforma administrativa integral y el proceso de regionalización. Santiago: Editora Nacional Gabriela Mistral,1976.

HUNEEUS, Carlos. El régimen de Pinochet. Santiago: Taurus, 2016.

JORDAN, Ricardo; VILLARROEL, Pablo. ¿Es Chile un País Regionalizado? Revista de Trabajo Social, Santiago, n. 57, 1990.

LIRA, Luis. La descentralización y el desarrollo regional: la experiencia chilena. Seminario Internacional Descentralización y Federalismo Fiscal organizado por ILPES entre el 2 al 13 de agosto de 2004.

MATTELARD, Armand. Atlas social de las comunas de Chile. Santiago: Pacífico, 1965. 
GARATE, Manuel. La revolución capitalista de Chile (1973-2003). Santiago: Universidad Alberto Hurtado, 2012.

RIFFO, Luis. Os espaços de globalização: a neoliberalização do território no Chile. SILVEIRA, Maria Laura (organizadora). Continente em chamas: globalização e território no América Latina. Rio de Janeiro: Civilização Brasileira, pp. 145-176, 2005.

SEVILLA, Álvaro. Hegemonía, gubernamentalidad, territorio. Apuntes metodológicos para una historia social de la planificación. Empiria. Revista de Metodología de Ciencias Sociales., Madrid, n. 27, pp. 49-72, 2014.

VALENZUELA, Esteban. Descentralización ya: conceptos, historia y agenda. Santiago: Ril, 2015. 